Research Article

\title{
Connectivity and Wiener Index of Fuzzy Incidence Graphs
}

\author{
Juanyan Fang, ${ }^{1}$ Irfan Nazeer, ${ }^{2}$ Tabasam Rashid $\mathbb{D}^{2},{ }^{2}$ and Jia-Bao Liu $\mathbb{D}^{3}$ \\ ${ }^{1}$ Institute of Information Technology \& Engineering Management, Tongling College, Tongling 244000, China \\ ${ }^{2}$ University of Management and Technology (UMT), Lahore, Pakistan \\ ${ }^{3}$ School of Mathematics and Physics, Anhui Jianzhu University, Hefei 230601, China \\ Correspondence should be addressed to Jia-Bao Liu; liujiabaoad@163.com
}

Received 9 December 2020; Revised 7 January 2021; Accepted 25 January 2021; Published 4 February 2021

Academic Editor: Ali Ahmad

Copyright (C) 2021 Juanyan Fang et al. This is an open access article distributed under the Creative Commons Attribution License, which permits unrestricted use, distribution, and reproduction in any medium, provided the original work is properly cited.

Connectivity is a key theory in fuzzy incidence graphs (FIGs). In this paper, we introduced connectivity index (CI), average connectivity index (ACI), and Wiener index (WI) of FIGs. Three types of nodes including fuzzy incidence connectivity enhancing node (FICEN), fuzzy incidence connectivity reducing node (FICRN), and fuzzy incidence connectivity neutral node (FICNN) are also discussed in this paper. A correspondence between WI and CI of a FIG is also computed.

\section{Introduction and Preliminaries}

Zadeh [1] presented the theory of fuzzy set (FS) to resolve complications in tackling with precariousness. Since then, the FS theory becomes a rich area in multiple disciplines, including mathematics, computer science, and signal processing. The theory of graphs has been considered to play a vital role in dealing with real-life situations. A graph is an easy way of expressing information, including the relationship between different objects. The objects are shown by nodes, and relations are represented by edges. In this paper, all graphs are finite, simple, without loops, and undirected. When there is a lack of certainty in the illustration of the objects and their association, we need to draw a fuzzy graph (FG) model. Zadeh's FS provided a productive ground for the theory of FGs which has been proposed by Rosenfeld [2]. In a graph, the strength of connectedness (SC) between any two vertices is either 0 or 1 , whereas in FG, it is a real number $\in \in[0,1]$. The study of FGs leads many scientists to contribute in this field, such as Yeh and Bang [3] studied the concept of FGs independently and discussed its applications in clustering analysis. Bhattacharya and Suraweera [4] discussed an algorithm to compute the max-min powers and property of FGs. Bhutani [5] worked on automorphism of FGs. Mordeson [6] introduced fuzzy line graphs. Bhutani and Rosenfeld $[7,8]$ studied strong arcs as well as fuzzy end nodes in FGs. Sunitha and Vijayakumar $[9,10]$ defined fuzzy trees and fuzzy blocks in FGs. Samanta et al. [11] inaugurated completeness and regularity of generalized FGs. Samanta and Pal [12] studied fuzzy planner graphs. Mathew and Sunitha [13] classified the edges of a FG as an $\alpha$-strong, $\beta$-strong, and $\delta$-edge. Mathew and Sunitha $[14,15]$ presented vertex, edge connectivity, and cycle connectivity in FGs. Mathew et al. [16] initiated saturation in FGs, and Binu et al. [17] explored CI and its application in FGs. Binu et al. [18] investigated CI of FG and its application to human trafficking. For some other significant works on graphs and FGs, one may refer to [19-25].

Wiener [26] was the first who investigated WI when he was studying about the boiling point of paraffin. After the landmark work of Harold Wiener about WI, in the middle of 1970s, new results related to WI were described. In graphs, WI has been studied in different fields such as Chemistry, Mathematics, and Physics. Binu et al. [27] discussed WI of FG and its application to illegal immigration networks.

FGs are unable to provide any information on the effect of a vertex on edges of the graph. Therefore, this disadvantage opens a way to introduce FIGs. FIGs talk about the effect of a vertex on an edge. Dinesh [28] presented the idea of FIGs. For example, if vertices show different residence societies and edges show roads joining these residence societies, we can have a FG expressing the extent of traffic from 
one society to another. The society has the maximum number of residents and will have maximum ramps in society. So, if $c$ and $d$ are two societies and $c d$ is a road joining them, then $(c, c d)$ could express the ramp system from the road $c d$ to the society $c$. In the case of an unweighted graph, $c$ and $d$ both will have an influence of 1 on $c d$. In a directed graph, the influence of $c$ on $c d$ represented by $(c, c d)$ is 1 , whereas $(d, c d)$ is 0 . This idea can be generalized by FIGs. Mordeson [29] studied numerous connectivity perceptions in FIGs. Malik et al. [30] explained different uses of FIGs. Mordeson et al. [31] proposed a fuzzy incidence (HTML translation failed) blocks along with their applications. Mordeson and Mathew [32] discussed different connectivity ideas in FIGs.

The motivation of our work is that CI, ACI, and WI of FGs exists in literature, but these indices are unknown for FIGs. These indices will make a way to study different properties of FIGs at length. This is why we propose these concepts for FIGs. Our work will open the new doors for many researchers to study FIGs in detail. The outline of this paper is as follows. In Section 1, we provide elementary definitions, results, and expressions of FIGs, which are required for the development of the content. In Section 2, we discuss CI of FIGs. Section 3 describes certain boundaries for CI of FIGs. CI of vertex and edge deleted fuzzy incidence subgraph (FIS) is illustrated in Section 4. Section 5 explains ACI and its characteristics. In Section 6, we discuss WI of FIG and a relationship between connectivity and WI. Below, we present some preliminary definitions from $[17,19,32]$.

Let $G$ be a simple graph with vertex set $V(G)$ and edge set $E(G)$. Then, an incidence graph (IG) is given by $G=(V$, $E, I)$, where $I \subseteq V \times E$. An IG is shown in Figure 1 , and if $(u, u v)$ is in IG, then $(u, u v)$ is said to be an incidence pair or pair. Assume an IG $G=(V, E, I)$. A sequence $v_{0},\left(v_{0}, v_{0} v_{1}\right)$, $v_{0} v_{1},\left(v_{1}, v_{0} v_{1}\right), v_{1}, \ldots, v_{n-1}, \quad\left(v_{n-1}, v_{n-1} v_{n}\right), v_{n-1} v_{n},\left(v_{n}, v_{n-1}\right.$ $\left.v_{n}\right), v_{n}$ is said to be a walk. It is closed if $v_{0}=v_{n}$. A walk is called a path if it has all distinct vertices. An IG is said to be connected if all pair of vertices are joined by a path. An edge $a b$ is said to be a fuzzy bridge (FB) if the deletion of $a b \in \theta^{*}$ lessens the SC between some pair of vertices in $G$.

In this paper, minimum is represented by $\wedge$ and $\max -$ imum is expressed by $\mathrm{V}$.

Definition 1. Consider a graph $G=(V, E)$, and $\eta$ and $\theta$ are fuzzy subsets of $V$ and $E$, respectively. Assume $V \times E$ has a fuzzy subset $\psi$. If $\psi\left(v^{*}, e^{*}\right) \leq \eta\left(v^{*}\right) \wedge \theta\left(e^{*}\right)$ for every $v^{*} \in V$ and $e^{*} \in E$, then $\psi$ is called a FI of $G$.

Definition 2. Assume a graph $G=(V, E)$ and $(\eta, \theta)$ be a fuzzy subgraph of $G$, if $\psi$ is a FI of $G$, then $G=(\eta, \theta, \psi)$ is called a FIG of $G$.

Definition 3. Consider a FIGG $=(\eta, \theta, \psi)$. Then, $H=(\kappa, \phi, \Omega)$ is a FIS of $G$ if $\kappa \subseteq \eta, \phi \subseteq \theta$ and $\Omega \subseteq \psi$.

Definition 4. Assume $G=(\eta, \theta, \psi)$ is a FIG. A FI path $\lambda$ from $g$ to $h, g, g h \in \eta^{*} \cup \theta^{*}$, is defined as a sequence of elements $\eta^{*}, \theta^{*}$, and $\psi^{*}$ beginning with $g$ and closing with $h$. The

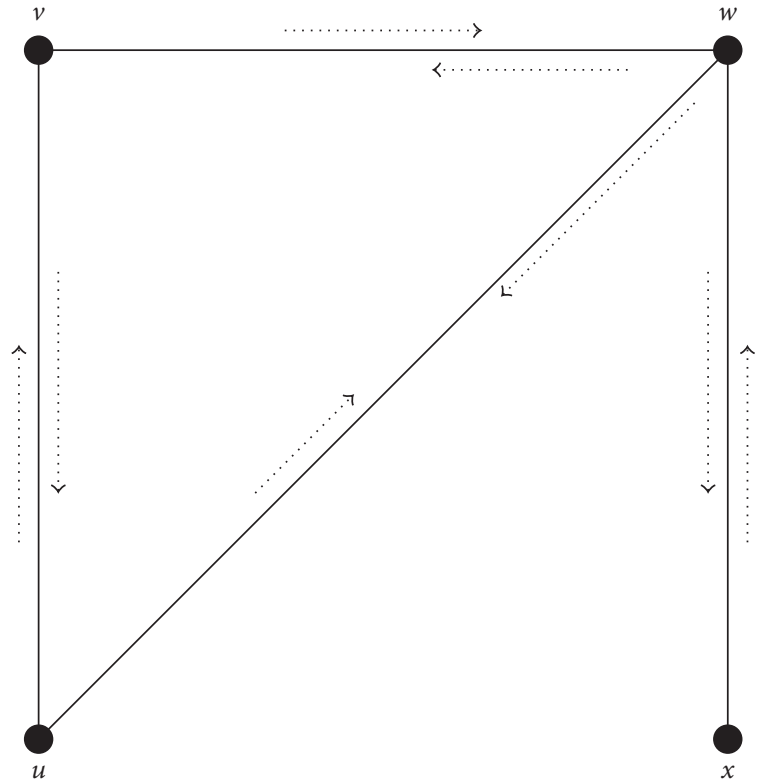

FIgURE 1: Incidence graph.

minimum value of $\psi(x, x y)$ is called incidence strength (IS), where $(x, x y) \in \lambda$.

In FIG, the incidence paths (IPs) can take distinct forms. $u_{0}=\left(u_{0}, u_{0} u_{1}\right), u_{0} u_{1}$ is an IP of length one. $u_{0}=\left(u_{0}\right.$, $\left.u_{0} u_{1}\right), u_{0} u_{1},\left(u_{1}, u_{0} u_{1}\right) u_{1}$ is an IP of length two.

Definition 5. Consider $G$ be a FIG. An incidence pair $(a, a b)$ is strong if $\psi(a, a b) \geq \operatorname{ICONN}_{G-(a, a b)}(a, a b)$ where $\operatorname{ICONN}_{G^{-}(a, a b)}(a, a b)$ shows the highest IS of $a-a b$. If $\psi(a, a b)>\mathrm{ICONN}_{G-(a, a b)}(a, a b)$, then the pair is called $\alpha$-strong. If $\psi(a, a b)=\operatorname{ICONN}_{G-(a, a b)}(a, a b)$, then this type of pair is $\beta$-strong. If an incidence pair is $\alpha$-strong or $\beta$-strong, then this kind of pair is a strong pair. If HTML translation failed, then this type of pair is called $\delta$ - incidence pair.

Definition 6. Assume $G$ be a FIG. If all pairs of $\lambda$ are strong, then an IP $\lambda$ in $G$ is called strong IP.

Definition 7. Consider $G=(\eta, \theta, \psi)$ be a FIG. $H=(\kappa, \phi, \Omega)$ is called a subgraph of $G$ if $\kappa(a)=\eta(a)$ for all $a \in \kappa^{*}$, $\phi(a b)=\theta(a b)$ for all $a b \in \phi^{*}$, and $\Omega(a, a b)=\psi(a, a b)$ for all pair $(a, a b) \in \Omega^{*}$.

Proposition 1. If $H$ is a FIS of $G$, then $\operatorname{ICONN}_{H(a, a b)}$ $\leq \operatorname{ICONN}_{G(a, a b)}$.

Definition 8. A FIG of $G$ is said to be complete if $\psi(a, a b)=$ $\eta(a) \wedge \theta(a b)$ for every $(a, a b) \in \psi^{*}$.

Definition 9 (see [19]). The distance $d(u, v)$ between two vertices $u, v \in V(G)$ is the minimum number of edges in a path between $u$ and $v$ in $G$. 
Definition 10. In a graph $G$, a path of shortest length is called geodesic.

Definition 11. (see [19]). WI of a graph $G$ is the sum of distances between all pairs of vertices of $G$. Then, the WI of a graph $G$ is given by $W(G)=\sum_{u, v \in V(G)} d(u, v)$.

\section{Connectivity Index of Fuzzy Incidence Graph}

Connectivity is a common parameter associated with a network. This section includes the introduction and formula to calculate CI of FIG. For easiness, in the coming sections, we will take $\eta(a)=1$ for every $a \in \eta^{*}$.

Definition 12. Let $G=(\eta, \theta, \psi)$ be a FIG. The $\mathrm{CI}$ of $G$ is given by

$$
\mathrm{CI}(G)=\sum_{a, b \in \eta^{*}} \eta(a) \eta(b) \operatorname{ICONN}_{G}(a, b),
$$

$\operatorname{ICONN}_{G}(a, b)$ is the maximum value of ISs for all the possible IPs between $a$ and $b$.

Example 1. Assume $G$ is a FIG given in Figure 2 having $\eta^{*}=\{i, j, k, l\} ; \quad \theta(i j)=0.8, \theta(i k)=0.4, \theta(j k)=0.7, \theta(k l)=$ $0.9 ; \psi(i, i j)=0.7, \psi(j, j i)=0.5, \psi(i, i k)=0.3, \psi(k, k i)=0.2$, $\psi(j, j k)=0.3, \psi(k, k j)=0.7, \psi(k, k l)=0.7$, and $\psi(l, l k)=$ 0.3 with $\mathrm{CI}(G)=2.0$.

The connectivity indices of subgraphs of FIGs can never be surpassed that of the FIGs. Therefore, a subgraph $H$ of FIG $G$ will have to be less than or equal to $\mathrm{CI}$ than the $\mathrm{CI}(G)$. This is shown in the coming proposition.

Proposition 2. If $H=(\kappa, \phi, \Omega)$ is a FIS of $G=(\eta, \theta, \psi)$, then $C I(H) \leq C I(G)$.

Proof. Let $a, b \in \kappa^{*}$. As $H=(\kappa, \phi, \Omega)$ is a FIS of $G=(\eta, \theta, \psi)$, $\kappa(a) \leq \eta(a)$. Also, if $H$ is a FIS of $G$, then $\operatorname{ICONN}_{H}(a, b) \leq$ $\operatorname{ICONN}_{G}(a, b)$ for any two $a, b \in \kappa^{*}$. This implies $\sum_{a, b \in \kappa^{*}} \kappa(a) \kappa(b) \operatorname{ICONN}_{H}(a, b) \leq \sum_{a, b \in \eta^{*}} \eta(a) \eta(b) \operatorname{ICONN}_{G}$ $(a, b)$ which implies $\mathrm{CI}(H) \leq \mathrm{CI}(G)$.

Example 2. Consider $G$ be a FIG provided in Figure 3 having $\eta^{*}=\{i, j, k\}, \quad \theta(i j)=0.8, \theta(i k)=0.4, \theta(j k)=0.7 ; \psi(i, i j)=$ $0.7, \psi(j, j i)=0.5, \psi(i, i k)=0.3, \psi(k, k i)=0.2, \psi(j, j k)=$ $0.3, \psi(k, k j)=0.7$. It is a subgraph of a FIG given in Example 1 (Figure 2) with CI 1.1.

\section{Bounds for Connectivity Index of Fuzzy Incidence Graph}

This section discusses some bounds for the CI of FIGs. Every FIG has a different CI. Therefore, all FIGs have different bounds for the CI. From all FIGs, the complete FIGs will have the highest CI. It is shown in the next theorem.

Theorem 1. Consider a FIG, G having $\left|\eta^{*}\right|=n$, then $0 \leq C I(G) \leq C I\left(G^{\prime \prime}\right)$, where $G^{\prime \prime}$ indicates a complete FIG.

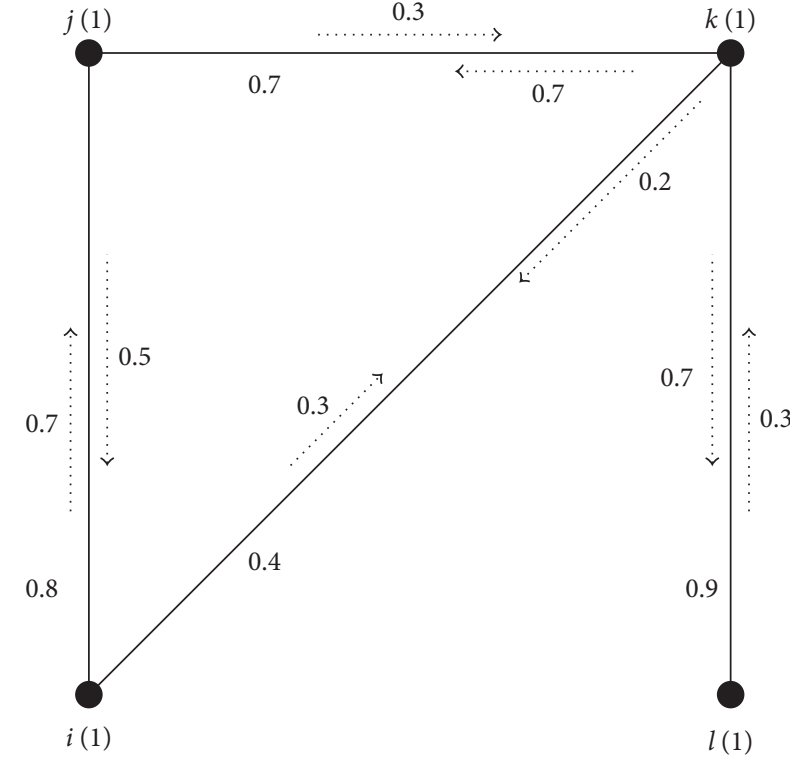

Figure 2: A fuzzy incidence graph with $\mathrm{CI}=2.0$.

Proof. Assume $G$ is a FIG. If $\left|\theta^{*}\right|=0$, then CI of $G=0$. Let $G^{\prime \prime}$ be the complete FIG having $\left|\eta^{*}\right|=n$ and $\eta^{\prime \prime}(a)=\eta(a)$. Then, $\quad \theta(a, b) \leq \theta^{\prime \prime}(a, b)$. Also, $\quad \operatorname{ICONN}_{G}(a, b) \leq$ $\operatorname{ICONN}_{G^{\prime \prime}}(a, b)$. This implies $0 \leq \mathrm{CI}(G) \leq \mathrm{CI}\left(G^{\prime \prime}\right)$.

Example 3. Assume $G^{\prime \prime}$ is a complete FIG given in Figure 4 having $\eta^{*}=\{i, j, k\}, \quad \theta(i j)=0.8, \theta(i k)=0.4, \theta(j k)=0.7$, $\psi(i, i j)=0.8, \psi(j, j i)=0.8, \psi(i, i k)=0.4, \psi(k, k i)=0.4, \psi$ $(j, j k)=0.7$ and $\psi(k, k j)=0.7$. We get $\mathrm{CI}\left(G^{\prime \prime}\right)=2.2$.

\section{Vertex-Deleted and Edge-Deleted Fuzzy Incidence Subgraphs with Connectivity Indices}

This section talks about the deletion of some edge or vertex of any FIG will become a cause of reducing a CI of FIG. An edge deleted and vertex deleted subgraph of a FIG will have small values of CI. The CI of FISs relies upon the nature of vertex or edge deleted.

Example 4. Let $G=(\eta, \theta, \psi)$ be the FIG given in Figure 5 with $\quad \eta^{*}=\{p, q, r, s, t\}, \quad \theta(p q)=0.7, \theta(p r)=0.5, \theta(q r)=$ $0.9, \theta(r s)=0.3, \theta(r t)=0.5, \theta(s t)=0.6 ; \psi(p, p q)=0.6, \psi \quad(q$, $q p)=0.7, \psi(p, p r)=0.4, \psi(r, r p)=0.2, \psi(q, q r)=0.8, \psi(r$, $r q)=0.6, \psi(r, r s)=0.2, \psi(s, s r)=0.3, \psi(r, r t)=0.5, \psi(t, t r)$ $=0.4, \psi(s, s t)=0.6$, and $\psi(t, t s)=0.4$. After calculation, we get $\mathrm{CI}(G)=4.6$, whereas $\mathrm{CI}(G-q r)=3.0$ and $\mathrm{CI}(G-$ $r t)=3.4$. Here, we conclude that deletion of some of the incidence pair reduces $\mathrm{CI}(G)$. This motivation leads us to the following result.

Theorem 2. Let $G=(\eta, \theta, \psi)$ be a FIG and $G^{*}=\left(\eta^{*}, \theta^{*}, \psi^{*}\right)$ be the FIS of $G$ by deleting an incidence pair $(a, a b) \in \psi^{*}$. Then, $C I\left(G^{*}\right)<C I(G)$ iff $(a, a b)$ is a FB. 


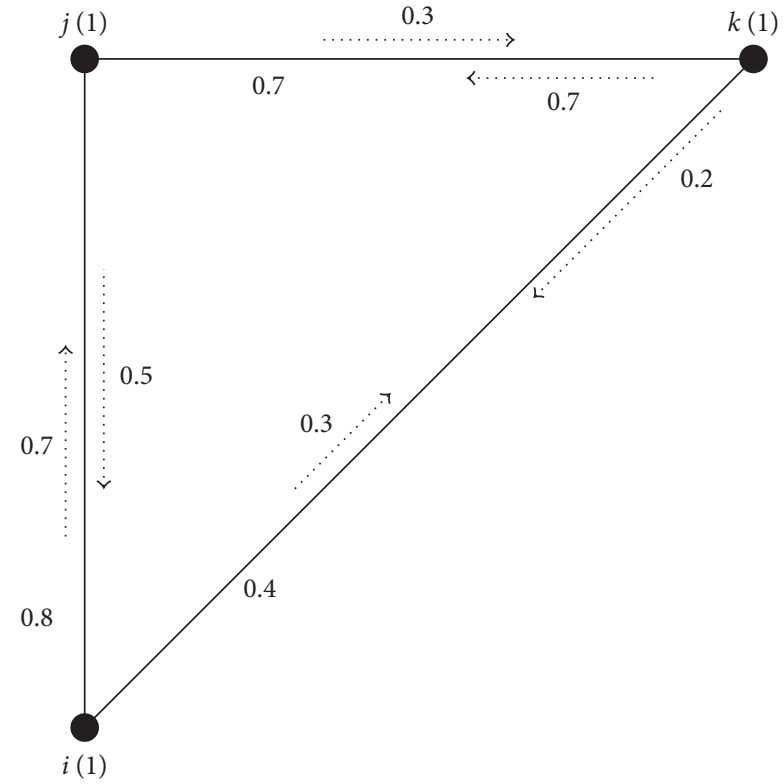

Figure 3: Subgraph of the fuzzy incidence graph in Figure 2.

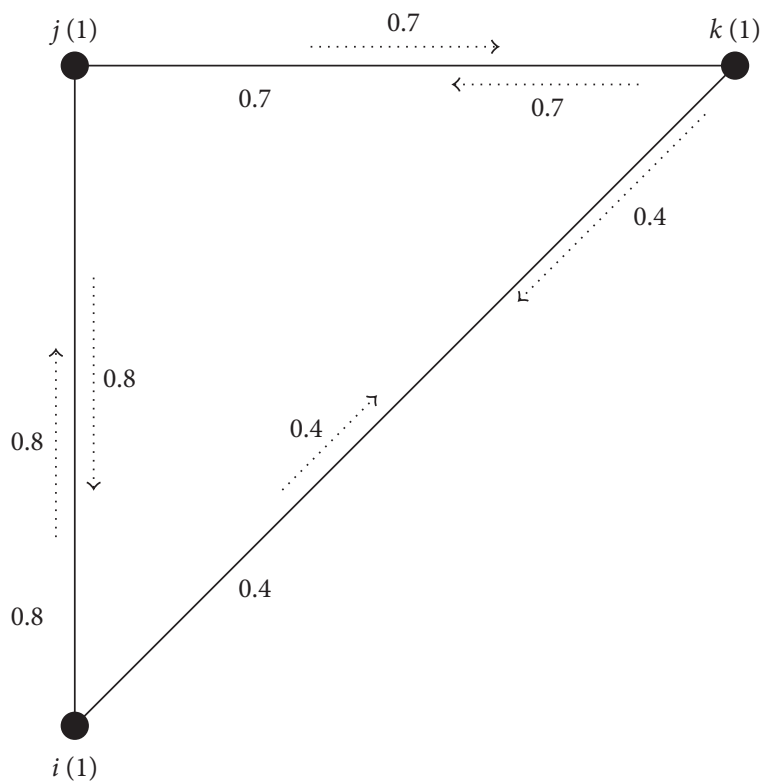

Figure 4: A complete fuzzy incidence graph.

Proof. Consider a pair $(a, a b)$ be a FB. Then, according to definition of FB, $\operatorname{ICONN}_{G-(a, a b)}(a, b)<\operatorname{ICONN}_{G}(a, b)$, which shows that $\mathrm{CI}\left(G^{*}\right)<\mathrm{CI}(G)$. Conversely, assume that $\mathrm{CI}\left(G^{*}\right)<\mathrm{CI}(G)$. Now, consider three different cases:

Case 1: consider a $\delta$-pair, $\psi(a, a b)$, and then $\psi(a, a b)<\operatorname{ICONN}_{G-(a, a b)}(a, b) \quad$ which implies $\operatorname{ICONN}_{G-(a, a b)}(a, b)<\operatorname{ICONN}_{G}(a, b)$. This implies $\mathrm{CI}\left(G^{*}\right)<\mathrm{CI}(G)$.

Case 2: consider a $\beta$-strong incidence pair $\psi(a, a b)$. Then, $\psi(a, a b)=\operatorname{ICONN}_{G-(a, a b)}(a, b)$ which means that there is an another strongest path $a,(a, a b)$, $a b,(b, a b), b$ different from the edge $\psi(a, a b)$. The IS

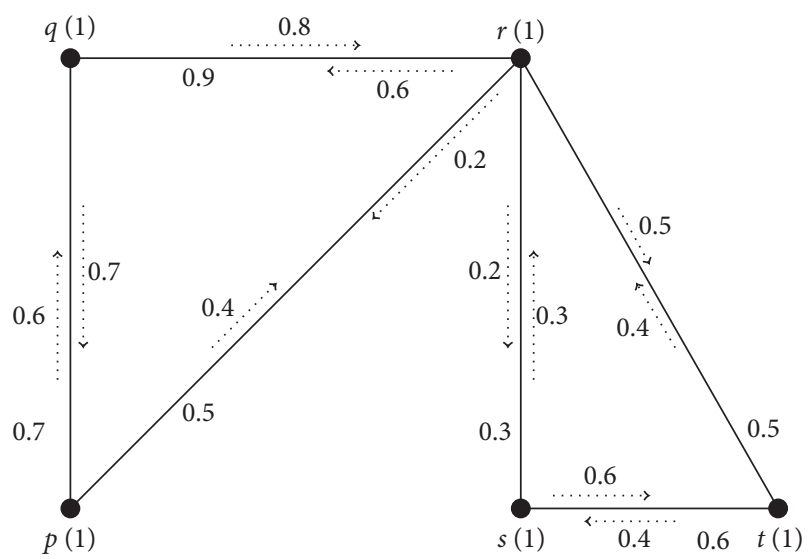

Figure 5: Fuzzy incidence graph with CI 4.6.

does not affect when we delete $\psi(a, a b)$. This means $\mathrm{CI}\left(G^{*}\right)=\mathrm{CI}(G)$.

Case 3: consider an $\alpha$-strong incidence pair $\psi(a, a b)$. Then, $\psi(a, a b)>\operatorname{ICONN}_{G-(a, a b)}(a, b)$. This means an edge $a b$ is the only strongest path whose strength is equal to $\psi(a, a b)$. From this, it is obvious that $\mathrm{CI}\left(G^{*}\right)<\mathrm{CI}(G)$. As by definition $\alpha$, strong arcs are FBs. This means if $\mathrm{CI}\left(G^{*}\right)<\mathrm{CI}(G)$, then $a b$ is a FB. This shows that $\mathrm{CI}\left(G^{*}\right)<\mathrm{CI}(G)$ iff $u v$ is a $\mathrm{FB}$.

\section{ACI of FIG}

We measure the average flow in the network to check how much flow is stable in the network. Therefore, in this section, we are going to introduce a new parameter named ACI of FIG. Assume the FIG given in Example 1.

Example 5. Assume $G$ is a FIG given in Example 1 (Figure 2) having $\eta^{*}=\{i, j, k, l\}, \theta(i j)=0.8, \theta(i k)=0.4, \theta(j k)=0.7$, $\theta(k l)=0.9 ; \psi(i, i j)=0.7, \psi(j, j i)=0.5, \psi(i, i k)=0.3, \psi(k$, $k i)=0.2, \psi(j, j k)=0.3, \psi(k, k j)=0.7, \psi(k, k l)=0.7 \quad$ and $\psi(l, l k)=0.3$ with $\mathrm{CI}(G)=2.0$. $G$ contains $4 ! /((4-2) ! 2 !)=$ 6 pairs of nodes with $\mathrm{ACI}(G)=\mathrm{CI}(G) / 6=2 / 6=0.33$.

Definition 13. Consider a FIG. The ACI of FIG is given by

$$
\operatorname{ACI}(G)=\frac{1}{\left(\begin{array}{c}
n \\
2
\end{array}\right)} \sum_{a, b \in \eta^{*}} \eta(a) \eta(b) \operatorname{ICONN}_{G}(a, b) .
$$

Definition 14. Assume a FIG and $z \in \eta^{*}$. $z$ will be FICRN of $G$ if $\operatorname{ACI}(G-z)<\operatorname{ACI}(G)$. We call $z$ a FICEN if ACI $(G-z)>\mathrm{ACI}(G) . z$ will be FICNN if $\mathrm{ACI}(G-z)=\mathrm{ACI}(G)$.

Example 6. Let $G$ be a FIG given in Figure 6 with (HTML translation failed), $\theta(i j)=0.3, \theta(i k)=0.8, \theta(j k)=$ $0.7, \theta(j n))=0.5, \theta(k l)=0.9, \theta(l m)=0.8 ; \psi(i, i j)=0.3, \psi(j$, $j i)=0.2, \psi(i, i k)=0.4, \psi(k, k i)=0.6, \psi(j, j k)=0.6, \psi(k$, $k j)=0.7, \psi(j, j n)=0.5, \psi(n, n j)=0.4, \psi(k, k l)=0.8, \psi(l$, $l k)=0.2, \psi(l, l m)=0.3$, and $\psi(m, m l)=0.7 . \quad \operatorname{ACI}(G)=$ 0.3 , ACI $(G-i)=0.29, \operatorname{ACI}(G-j)=0.25$, ACI $(G-k)=$ 


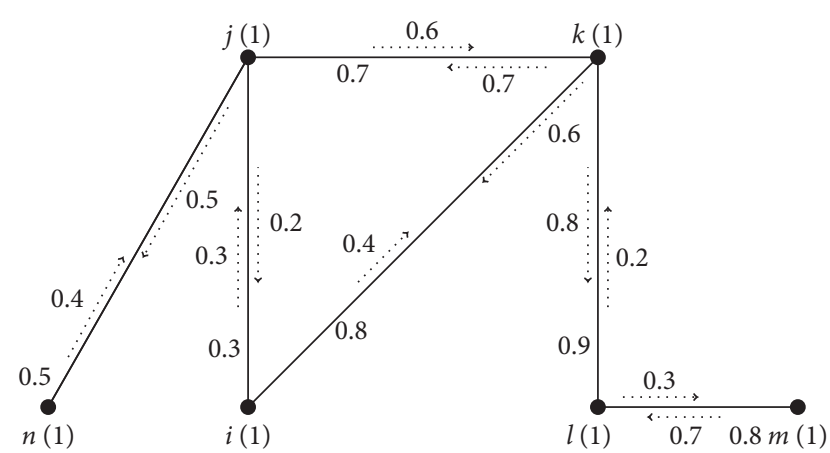

Figure 6: Fuzzy incidence graph with FICRNs and FICENs.

$0.11, \mathrm{ACI}(G-l)=0.36, \mathrm{ACI}(G-m)=0.34, \mathrm{ACI}(G-n)=$ 0.29. Therefore, $i, j, k$, and $n$ are FICRNs; $l$ and $m$ are FICENs.

In the following proposition with the help of $\mathrm{CI}$, we classify these nodes:

Proposition 3. Consider a FIG. Let $z \in \eta^{*}$ having $n=\left|\eta^{*}\right| \geq 3$. Assume $q=C I(G) / C I(G-m)$. $z$ is a FICEN iff $q<(n /(n-2)) . z$ is a FICRN iff $q>n /(n-2) . z$ is a FICNN iff $q=n /(n-2)$.

Proof. Let $z$ be FICNN of FIG. Then, ACI $(G)=\mathrm{ACI}(G-z)$, that is, $\mathrm{CI}(G) /\left(\begin{array}{l}n \\ 2\end{array}\right)=\mathrm{CI}(G-z) /\left(\begin{array}{c}n-1 \\ 2\end{array}\right)$ and $\mathrm{CI}(G)$ $/ \mathrm{CI}(G-z)=\left(\begin{array}{l}n \\ 2\end{array}\right) /\left(\begin{array}{c}n-1 \\ 2\end{array}\right), \quad$ which implies $\mathrm{CI}(G) / \mathrm{CI}(G-z)=n /(n-2)$. By reversing the argument, the sufficient part can easily be proved. In similar manners, the other two cases can be solved.

Definition 15. Let $G$ be a FIG. $G$ will be FI connectivity enhancing graph if $G$ contains minimum one FICEN. If $G$ contains no FICEN and has minimum one FICRN, then we call it FI connectivity reducing graph. We call $G$ a FI connectivity neutral graph if all nodes of $G$ are neutral.

\section{Wiener Index of FIG}

In this section, we establish the concept of WI. WI is a topological index used in different fields like medicine, communication, and cryptology. A proper definition to calculate WI of FIG is given next.

Definition 16. Let $G$ be a FIG. WI of $G$ can be calculated as

$$
\mathrm{WI}(G)=\sum_{a, b \in \eta^{*}} \eta(a) \eta(b) d_{s}(a, b)
$$

where $d_{s}(a, b)$ represents weights of those strong geodesics from $a-b$ whose sum is minimum.

Example 7. Consider $G$ be a FIG given in Figure 7 with $\eta^{*}=\{i, j, k\}, \quad \theta(i j)=0.7, \theta(i k)=0.8, \theta(j k)=0.7 ; \psi(i, i j)=$ $0.6, \psi(j, j i)=0.6, \psi(i, i k)=0.8, \psi(k, k i)=0.8, \psi(j, j k)=$

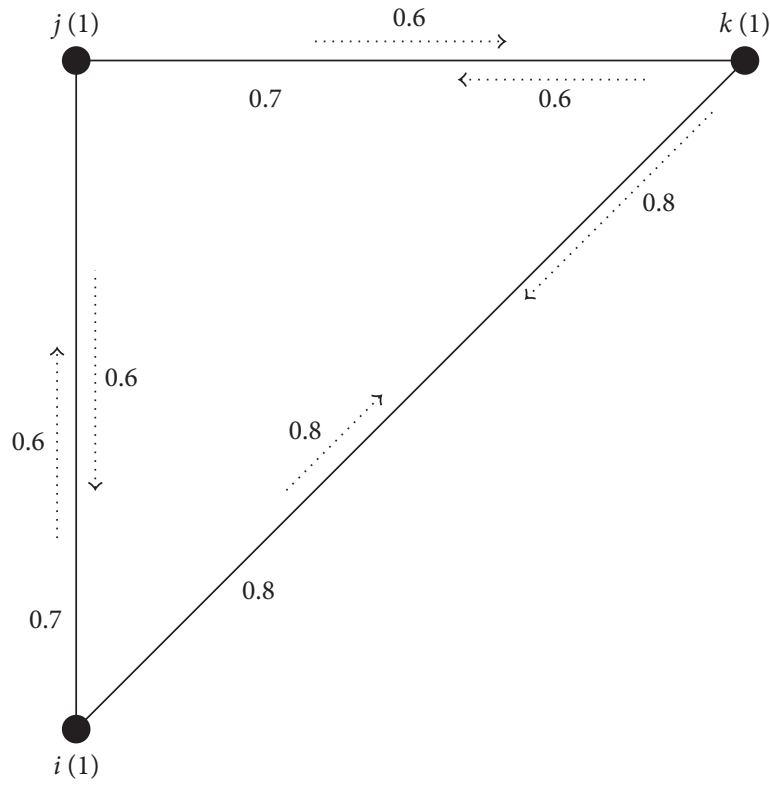

Figure 7: Fuzzy incidence graph with $\mathrm{WI}(G)=4$.

$0.6, \psi(k, k j)=0.6$. Here, each incidence pair is a strong pair. Because $i-j$ is a geodesic, it is a strongest path from $i-j$. Similarly, $i-k$ and $j-k$ are all strongest paths. Thus, $\mathrm{WI}(G)=4$.

Assume a FIG. Let $H$ be the FIS of $G$. Then, it is not necessary that $\mathrm{WI}(H) \leq \mathrm{WI}(G)$. It can be seen in the next example

Example 8. Let $H=(\kappa, \phi, \Omega)$ is a FIS (see Figure 8) of $G=$ $(\eta, \theta, \psi)$ (in Figure 7) such that $\phi(i k)=0$ and $\phi(i j)=$ $0.7, \phi(j k)=0.7, \Omega(i, i j)=0.6, \Omega(j, i j)=0.6, \Omega(j, j k)=0.6$, $\Omega(k, j k)=0.6$. Geodesic from $i$ to $k$ is $i, j, k$. $d_{s}(i, j)=$ $\psi(i, i j)+\psi(j, i j)=1.2$ Similarly, $d_{s}(i, k)=2.4, d_{s}(j, k)=$ 1.2. Then, $\mathrm{WI}(H)=4.8$ and $\mathrm{WI}(G)=4<\mathrm{WI}(H)=4.8$.

Link between WI and CI of a FIG

In FIGs, it could be noted that CI will be less than WI.

Example 9. Consider a FIG given in Figure 9 with $\eta^{*}=$ $\{i, j, k, l\}, \quad \theta(i j)=0.8, \theta(i k)=0.4, \theta(j k)=0.7, \theta(k l)=0.9$; $\psi(i, i j)=0.5, \psi(j, j i)=0.7, \psi(i, i k)=0.3, \psi(k, k i)=0.2, \psi$ $(j, j k)=0.3, \psi(k, k j)=0.7, \psi(k, k l)=0.7$, and $\psi(l, l k)=0.3$. This FIG contains each pair strong except $\psi(i, k i)=0.2$ because $\operatorname{ICONN}_{G-(i, k i)}=0.3$. For $(i, k) \in \eta^{*} \times \eta^{*} \backslash \psi(i, k i)$. Now, $\quad \mathrm{CI}(G)=0.5+0.3+0.3+0.3+0.3+0.3=2 \quad$ and $\mathrm{WI}(G)=1.2+2.2+3.2+1+2+1=10.6$ Thus, $\mathrm{CI}(G)<$ $\mathrm{WI}(G)$.

Theorem 3. Assume a FIG having $\left|\eta^{*}\right| \geq 3$. Then, $W I(G)>C I(G)$.

Proof. Consider a FIG with $\left|\eta^{*}\right| \geq 3$. For every $a, b \in \eta^{*}$, the sum of membership values of every strong incidence pairs connecting $a$ and $b$ is $d_{s}(a, b)$, whereas the minimum membership value of all strong incidence pairs is 


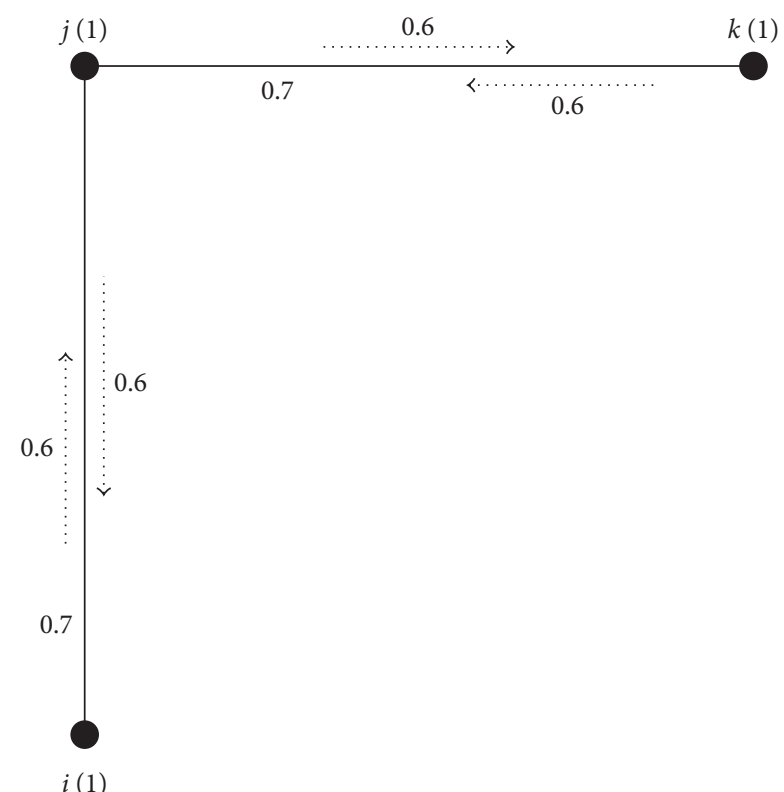

Figure 8: Fuzzy incidence subgraph of Figure 7.

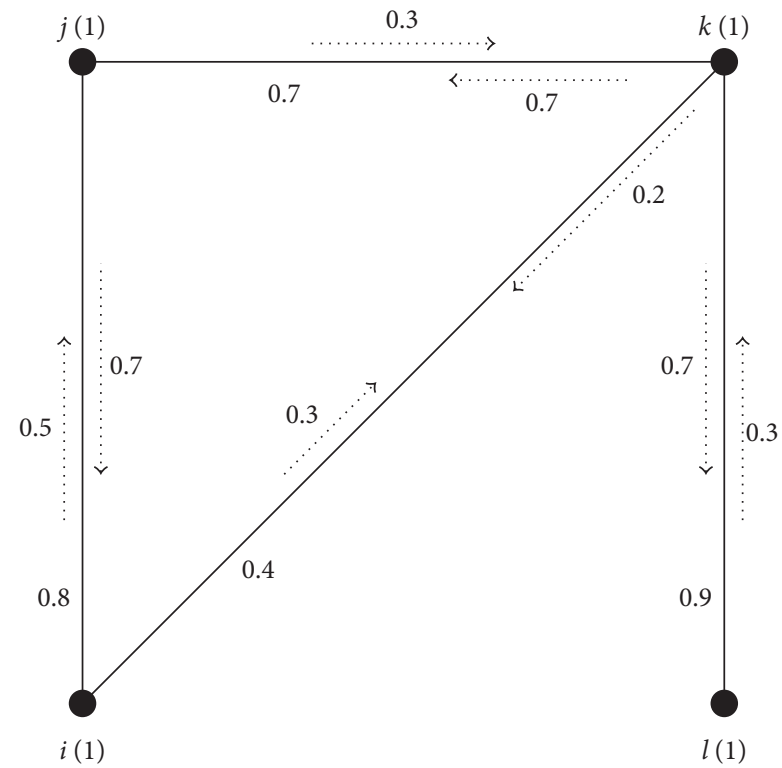

Figure 9: Fuzzy incidence graph with $\mathrm{CI}<\mathrm{WI}$.

$\operatorname{ICONN}_{G}(a, b)$, which means $\operatorname{ICOON}_{G}(a, b)<d_{s}(a, b)$. This implies $\quad \sum_{a, b \in \eta^{*}} \eta(a) \eta(b) \operatorname{ICONN}_{G}(a, b)<\sum_{a, b \in \eta^{*}} \eta(a) \eta$ $(b) d_{s}(a, b)$. Hence, $\mathrm{CI}(G)<\mathrm{WI}(G)$.

Theorem 4. Assume a complete FIG, $G$ with $\left|\eta^{*}\right|=2$, and $(a)=(b)=1$. Then, $2 C I(G)=W I(G)$.

Proof. Consider $G$ be a complete FIG with $\left|\eta^{*}\right|=2$ and $(a)=(b)=1$. $\exists$ There exist a strong path $P^{\prime \prime}$ which is the strongest path in complete FIG, G. For any two nodes $a, b \in \eta^{*}$, the sum of membership values of incidence pair in the only strongest path $P^{\prime \prime}$ connecting $a$ with $b$ is $d_{s}(a, b)$, whereas the minimum membership value of incidence pair

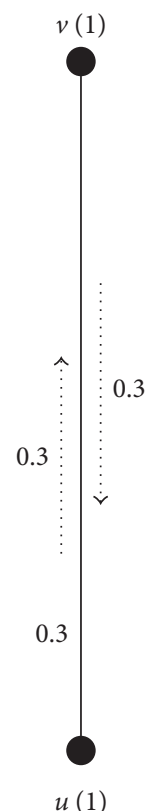

FIGURE 10: Complete fuzzy incidence graph with $2 \mathrm{CI}(G)=\mathrm{WI}(G)$.

of $P$ is $\operatorname{ICONN}_{G}(a, b)$. This implies $2 \operatorname{ICONN}_{G}(a, b)=d_{s}(a, b) . \quad$ Thus, $\quad 2 \sum_{a, b \in \eta^{*}} \eta$ $(a) \eta(b) \operatorname{ICONN}_{G}(a, b)=\sum_{a, b \in \eta^{*}} \eta(a) \eta(b) d_{s}(a, b)$. Hence, $2 \mathrm{CI}(G)=\mathrm{WI}(G)$.

Example 10. Let $G$ be the complete FIG, as shown in Figure 10. Here, $u, v \in \eta^{*}$ and $\theta(u v)=0.3, \psi(u, u v)=$ $0.3, \psi(v, v u)=0.3$. Clearly, $\mathrm{CI}(G)=0.3$ and $\mathrm{WI}(G)=0.6$.

\section{Conclusion}

Connectivity is an essential parameter attached to a network. The idea of connectivity is inseparable from the theory of FIGs. In this paper, we have come up with different results about WI and CI of FIGs. Relevant examples related to WI and CI of FIGs are too obtained. In this article, CI, ACI, and WI of FIGs linked with networks are expressed. Nodes of FIGs are classified as FICRN, FICEN, and FICNN by using these incidences. Various types of FIGs are also obtained. A crucial relationship between CI and WI of FIG is derived too. Our objective is to enlarge our research work to soft FIGs, bipolar FIGs, threshold FIGs, competition FIGs, regular FIGs, and $q$-rung FIGs. More similar results and applications will be reported in upcoming papers.

\section{Data Availability}

No data were used to support this study.

\section{Conflicts of Interest}

The authors declare that there are no conflicts of interest regarding the publication of this paper. 


\section{Acknowledgments}

This paper was supported by the Anhui Natural Science Research Project (2017) under Grant no. KJ2017A469 and Top-Notch Talents Cultivation Project (gxgnfx2020099) of Anhui Higher Education.

\section{References}

[1] L. A. Zadeh, "Fuzzy sets," Information and Control, vol. 8, no. 3, pp. 338-353, 1965.

[2] A. Rosenfeld, "Fuzzy graphs," in Fuzzy Sets and Their Applications, L. A. Zadeh, K. S. Fu, and M. Shimura, Eds., pp. 77-95, Academic Press, New York, NY, USA, 1975.

[3] R. T. Yeh and S. Y. Bang, "Fuzzy relations, fuzzy graphs and their applications to clustering analysis," in Fuzzy Sets and Their Applications, L. A. Zadeh, K. S. Fu, and M. Shimura, Eds., pp. 125-149, Academic Press, Cambridge, MA, USA, 1975.

[4] P. Bhattacharya and F. Suraweera, "An algorithm to compute the supremum of max-min powers and a property of fuzzy graphs," Pattern Recognition Letters, vol. 12, no. 7, pp. 413-420, 1991.

[5] K. R. Bhutani, "On automorphisms of fuzzy graphs," Pattern Recognition Letters, vol. 9, no. 3, pp. 159-162, 1989.

[6] J. N. Mordeson, "Fuzzy line graphs," Pattern Recognition Letters, vol. 14, no. 5, pp. 381-384, 1993.

[7] K. R. Bhutani and A. Rosenfeld, "Fuzzy end nodes in fuzzy graphs," Information Sciences, vol. 152, pp. 323-326, 2003.

[8] K. R. Bhutani and A. Rosenfeld, "Strong arcs in fuzzy graphs," Information Sciences, vol. 152, pp. 319-322, 2003.

[9] M. S. Sunitha and A. Vijayakumar, "A characterization of fuzzy trees," Information Sciences, vol. 113, no. 3-4, pp. 293-300, 1999 .

[10] M. S. Sunitha and A. Vijayakumar, "Blocks in fuzzy graphs," The Journal of Fuzzy Mathematics, vol. 13, no. 1, pp. 13-23, 2005.

[11] S. Samanta, B. Sarkar, D. Shin, and M. Pal, "Completeness and regularity of generalized fuzzy graphs," Springer Plus, vol. 5, no. 1, p. 1979, 2016.

[12] S. Samanta and M. Pal, "Fuzzy planar graphs," IEEE Transactions on Fuzzy Systems, vol. 23, no. 6, pp. 1936-1942, 2015.

[13] S. Mathew and M. S. Sunitha, "Types of arcs in a fuzzy graph," Information Sciences, vol. 179, no. 11, pp. 1760-1768, 2009.

[14] S. Mathew and M. S. Sunitha, "Node connectivity and arc connectivity of a fuzzy graph," Information Sciences, vol. 180, no. 4, pp. 519-531, 2010.

[15] S. Mathew and M. S. Sunitha, "Cycle connectivity in fuzzy graphs," Journal of Intelligent \& Fuzzy Systems, vol. 24, no. 3, pp. 549-554, 2013.

[16] S. Mathew, H. L. Yang, and J. K. Mathew, "Saturation in fuzzy graphs," New Mathematics and Natural Computation, vol. 14, no. 01, pp. 113-128, 2018.

[17] M. Binu, S. Mathew, and J. N. Mordeson, "Connectivity index of a fuzzy graph and its application to human trafficking," Fuzzy Sets and Systems, vol. 360, pp. 117-136, 2019.

[18] M. Binu, S. Mathew, and J. N. Mordeson, "Connectivity index of a fuzzy graph and its application to human trafficking," Fuzzy Sets Systems, vol. 360, pp. 117-136, 2019.

[19] N. Jicy and S. Mathew, "Some new connectivity parameters for weighted graphs," Journal of Uncertainty in Mathematics Science, vol. 2014, pp. 1-9, 2014.
[20] S. Mathew and M. S. Sunitha, "Some connectivity concepts in weighted graphs," Advances and Applications in Discrete Mathematics, vol. 6, no. 1, pp. 45-54, 2010.

[21] S. Mathew, J. N. Mordeson, and D. Malik, Fuzzy Graph Theory, Springer, Berlin, Germany, 2018.

[22] J. N. Mordeson, S. Mathew, and D. Malik, Fuzzy Graph Theory with Applications to Human Trafficking, Springer, Berlin, Germany, 2018.

[23] J. N. Mordeson and S. Mathew, "Human trafficking: source, transit, destination designations," New Mathematics and Natural Computation, vol. 13, no. 3, pp. 209-218, 2017.

[24] J. N. Mordeson, S. Mathew, and R. A. Borzooei, "Vulnerability and government response to human trafficking: vague graphs," New Mathematics and Natural Computation, vol. 14, no. 2, pp. 202-219, 2018.

[25] S. Samanta, M. Pal, H. Rashmanlou, and R. A. Borzooei, "Vague graphs and strengths," Journal of Intelligent \& Fuzzy Systems, vol. 30, no. 6, pp. 3675-3680, 2016.

[26] H. Wiener, "Structural determination of paraffin boiling points," Journal of the American Chemical Society, vol. 69, no. 1, pp. 17-20, 1947.

[27] M. Binu, S. Mathew, and J. N. Mordeson, "Wiener index of a fuzzy graph and application to illegal immigration networks," Fuzzy Sets and Systems, vol. 384, pp. 132-147, 2019.

[28] T. Dinesh, "Fuzzy incidence graph-an introduction," $A d$ vances in Fuzzy Sets and Systems, vol. 21, no. 1, pp. 33-48, 2016.

[29] J. N. Mordeson, "Fuzzy incidence graphs," Advances in Fuzzy Sets and Systems, vol. 21, no. 2, pp. 121-133, 2016.

[30] D. Malik, J. N. Mordeson, and S. Mathew, "Fuzzy incidence graphs: applications to human trafficking," Information Sciences, vol. 447, pp. 244-255, 2018.

[31] J. N. Mordeson and S. Mathew, "Fuzzy incidence blocks and their applications in illegal migration problems," New Mathematics and Natural Computation, vol. 13, pp. 245-260, 2017.

[32] S. Mathew and J. N. Mordeson, "Connectivity concepts in fuzzy incidence graphs," Information Sciences, vol. 382-383, pp. 326-333, 2017. 